\title{
Statistical and bioinformatic analysis of hemimethylation patterns in non-small cell lung cancer
}

\author{
Shuying Sun ${ }^{1 *} \mathbb{D}$, Austin Zane ${ }^{2}$, Carolyn Fulton ${ }^{3}$ and Jasmine Philipoom ${ }^{4}$
}

\begin{abstract}
Background: DNA methylation is an epigenetic event involving the addition of a methyl-group to a cytosineguanine base pair (i.e., CpG site). It is associated with different cancers. Our research focuses on studying non-small cell lung cancer hemimethylation, which refers to methylation occurring on only one of the two DNA strands. Many studies often assume that methylation occurs on both DNA strands at a CpG site. However, recent publications show the existence of hemimethylation and its significant impact. Therefore, it is important to identify cancer hemimethylation patterns.

Methods: In this paper, we use the Wilcoxon signed rank test to identify hemimethylated CpG sites based on publicly available non-small cell lung cancer methylation sequencing data. We then identify two types of hemimethylated $\mathrm{CpG}$ clusters, regular and polarity clusters, and genes with large numbers of hemimethylated sites. Highly hemimethylated genes are then studied for their biological interactions using available bioinformatics tools.

Results: In this paper, we have conducted the first-ever investigation of hemimethylation in lung cancer. Our results show that hemimethylation does exist in lung cells either as singletons or clusters. Most clusters contain only two or three $\mathrm{CpG}$ sites. Polarity clusters are much shorter than regular clusters and appear less frequently. The majority of clusters found in tumor samples have no overlap with clusters found in normal samples, and vice versa. Several genes that are known to be associated with cancer are hemimethylated differently between the cancerous and normal samples. Furthermore, highly hemimethylated genes exhibit many different interactions with other genes that may be associated with cancer. Hemimethylation has diverse patterns and frequencies that are comparable between normal and tumorous cells. Therefore, hemimethylation may be related to both normal and tumor cell development.
\end{abstract}

Conclusions: Our research has identified CpG clusters and genes that are hemimethylated in normal and lung tumor samples. Due to the potential impact of hemimethylation on gene expression and cell function, these clusters and genes may be important to advance our understanding of the development and progression of nonsmall cell lung cancer.

Keywords: Methylation, Hemimethylation, Lung Cancer, Bioinformatics, Epigenetics

\footnotetext{
* Correspondence: s_s355@txstate.edu

${ }^{1}$ Department of Mathematics, Texas State University, San Marcos, TX, USA

Full list of author information is available at the end of the article
}

C C The Author(s). 2021 Open Access This article is licensed under a Creative Commons Attribution 4.0 International License, which permits use, sharing, adaptation, distribution and reproduction in any medium or format, as long as you give appropriate credit to the original author(s) and the source, provide a link to the Creative Commons licence, and indicate if changes were made. The images or other third party material in this article are included in the article's Creative Commons licence, unless indicated otherwise in a credit line to the material. If material is not included in the article's Creative Commons licence and your intended use is not permitted by statutory regulation or exceeds the permitted use, you will need to obtain permission directly from the copyright holder. To view a copy of this licence, visit http://creativecommons.org/licenses/by/4.0/ The Creative Commons Public Domain Dedication waiver (http://creativecommons.org/publicdomain/zero/1.0/) applies to the data made available in this article, unless otherwise stated in a credit line to the data. 


\section{Background}

Lung cancer is a leading cause of death in the United States; more patients die of lung cancer than of breast, prostate, and colon cancers combined. The American Cancer Society predicts that in 2021 alone there will be 235,760 new cases of lung cancer diagnosed and 131,880 deaths in the United States [1]. The five-year survival rate of lung cancer is much lower than many other prominent cancers such as breast, colorectal, and prostate, as only $19.4 \%$ of patients survive beyond 5 years of having the disease. The rate of survival can be as high as $57.4 \%$ when the cancer is still localized. However, the majority (57\%) of patients are diagnosed in late stages, and the five-year survival rate of these patients is only $5.2 \%[2]$.

In order to diagnose and treat lung cancer, it is important to identify genetic and epigenetic biomarkers. One important epigenetic biomarker or event is DNA methylation. It is the covalent bonding of a methyl group $\left(-\mathrm{CH}_{3}\right)$ to a $\mathrm{CpG}$ site in a mammalian cell; this is an epigenetic alteration to the DNA, meaning the DNA sequence does not change. A CpG site is the nucleotide base cytosine bonded to the base guanine by a phosphate (5'-CpG-3') [3]. The correlation between methylation patterns on specific $\mathrm{CpG}$ sites and gene expression has been studied as methylation enhances or mutes particular genes [4]. DNA methylation patterns are maintained and changed mainly through two dynamic processes: methylation maintenance and de novo methylation [5, 6]. Methylation maintenance allows for preservation of methylation patterns across replication generations, maintaining valuable methylation levels. De novo methylation occurs on symmetrically unmethylated CpG sites and increases methylation levels over cell generations [5]. Demethylation is the action of a methyl group being removed from a CpG site, and it can be observed in two forms: passive and active [6]. Passive demethylation is an error during maintenance methylation, resulting in bare or hypomethylated $\mathrm{CpG}$ sites on the nascent strand, whilst the parent strand is methylated. In contrast, active demethylation is the deliberate removal of a methyl group from a CpG [7].

Both demethylation and de novo methylation can lead to the development of hemimethylation, i.e., methylation occurring on only one DNA strand of a CpG site but not the other. Because demethylation and de novo methylation are related to the loss and gain of methylation respectively, hemimethylation may be associated with the changes of methylation patterns and levels as cancer progresses [7]. That is, hemimethylation may be closely related to abnormal hypermethylation and hypomethylation patterns found in a cancer genome. In fact, Ehrlich and Lacey find that the study of hemimethylation provides valuable insight into cancer-associated active demethylation and hypomethylation [5]. Exactly how different hemimethylation patterns affect gene expression is not yet well documented, except for the recent findings by $\mathrm{Xu}$ and Corces, who show that the elimination of hemimethylation can reduce chromatic interactions [8]. They also show that in inner cell mass cells, there are a large number of hemimethylated CpG sites on gene bodies. These hemimethylated sites play a pleiotropic role on gene expression [8].

DNA methylation patterns and levels can vary as cancer progresses [7]. Abnormal hypermethylation and hypomethylation are commonly known characteristics of cancerous cells. Identification of these different states of methylation can assist in the detection of cancerous cells long before they would appear in clinical examinations or before symptoms are apparent. Hemimethylation as a transitional state or indicator of hypomethylation and hypermethylation can help medical researchers to monitor how far the disease has progressed. Knowledge of the hemimethylation can allow for better comprehension of certain cancers and provide better insight toward the development of treatment methods. Therefore, it is important to investigate it in cancer. Hemimethylation has been researched previously for breast cancer cell lines [9], but not yet for lung cancer. Lung cancer is a great candidate for this investigation as it is challenging to detect early-stage lung cancer. Its symptoms are often obscure or mistakable due to the consequence of previous smoking habits. Utilizing hemimethylated genes to identify carcinogenic development may be beneficial in lung cancer diagnosis. The purpose of this research study is to identify hemimethylation patterns in both normal and tumorous samples of non-small cell lung cancer patients using publicly available methylation sequencing data.

\section{Methods}

In this study, to identify hemimethylation patterns, we will analyze the methylation sequencing data generated from tumor and adjacent normal tissues of 18 male nonsmall cell lung cancer patients in their fifties to seventies. The reduced representation bisulfite sequencing (RRBS) datasets of these patients are publicly available [10]. Sequencing reads are aligned to the hg38 reference genome, and methylation signals are obtained using the BRAT-bw software package [11]. All methylation datasets consist of the methylation signals of CpG sites. These methylation levels are calculated based on the original or raw number of reads, that is, the methylation level or ratio at each CpG site is calculated as the number of methylated reads divided by total number of reads. Further analysis is then performed on CpG sites with at least four methylation signals for both strands.

Hemimethylation is a particular kind of methylation pattern. If a CpG is methylated on the forward strand 
but not on the reverse strand, it is defined as a MU hemimethylation site. If a $\mathrm{CpG}$ is methylated on the reverse strand but not on the forward strand, it is defined as a UM hemimethylation site. If a CpG exhibits no significant hemimethylation, it is defined as an NS site. If no data is available to be analyzed at a $\mathrm{CpG}$, that site is defined as an NA site. Hemimethylation occurs not only at solitary CpG sites, but also at consecutive ones, known as hemimethylation clusters. Such clusters can manifest in one of two distinct patterns: regular or polar $[5,7,9,12]$, see Fig. 1 . A regular cluster can be observed when sequential $\mathrm{CpG}$ sites are methylated on the same strand but unmethylated on the other. A polar or polarity cluster occurs when consecutive $\mathrm{CpG}$ sites are methylated on opposite strands. Next, we will explain in detail how to identify these different hemimethylation patterns.

The Wilcoxon signed rank test is utilized to investigate if hemimethylation exists at each CpG site $[9,13]$. This particular test is selected instead of a regular statistical ttest because the independence and normality assumptions are not satisfied. The null hypothesis is that, at each $\mathrm{CpG}$ site, there is no methylation level difference between the forward (or positive) and reverse (or negative) strands. For every CpG site, there are two methylation signals, one from the forward and one from the reverse strand. That is, there are up to 18 pairs of methylation signals at each CpG site as there are 18 samples. For each pair, the forward strand methylation level is subtracted from the reverse strand methylation level. The absolute value of the difference and the sign of the difference (negative or positive) are recorded separately. Pairs with zero difference are discarded. The absolute differences of the pairs are then ranked from smallest to largest so that the pair with the smallest absolute difference is ranked one. Lastly, a test statistic is calculated by summing all of the ranks after multiplying them by their respective signs (i.e., signed-ranks). This test statistic follows a specific distribution, and it is evaluated using a reference table. If the test statistic falls into the rejection region that is determined by the critical value from the table, then the null hypothesis is rejected. The rejection decision means that there is difference between the forward and reverse methylation levels at a CpG site. If the null hypothesis is not rejected, it shows that there is not a significant difference. The Wilcoxon signed rank test is conducted for tumor and normal samples separately. That is, we identify hemimethylated CpG sites for tumor and normal samples separately and then compare them.

The test results are filtered based on two metrics: forward and reverse strand methylation mean difference and Wilcoxon test $p$-value. CpG sites with a large mean difference in methylation levels and a $p$-value that is less than 0.05 are identified as hemimethylated CpG sites. Significant CpG sites are annotated to show which gene promoter region or gene body they belong to. Additionally, clusters of $\mathrm{CpG}$ sites are identified as either regular or polar patterns. For example, MMM-UUU and MU$\mathrm{UM}$ are regular and polar clusters respectively, see Fig. 1. MMM-UUU means that at three consecutive CPG sites, methylation occurs on the positive strands (i.e., MMM) but not on the reverse strand (i.e., UUU). MUUM means at two consecutive CpG sites, on the positive strand they are methylated $(\mathrm{M})$ and unmethylated $(\mathrm{U})$ respectively (i.e., MU), and on the reverse strand they are unmethylated $(\mathrm{U})$ and methylated $(\mathrm{M})$ respectively (i.e., UM). The CpG sites that are not in clusters are called singletons. The lengths of all clusters in tumor and normal cells are shown in histograms. The

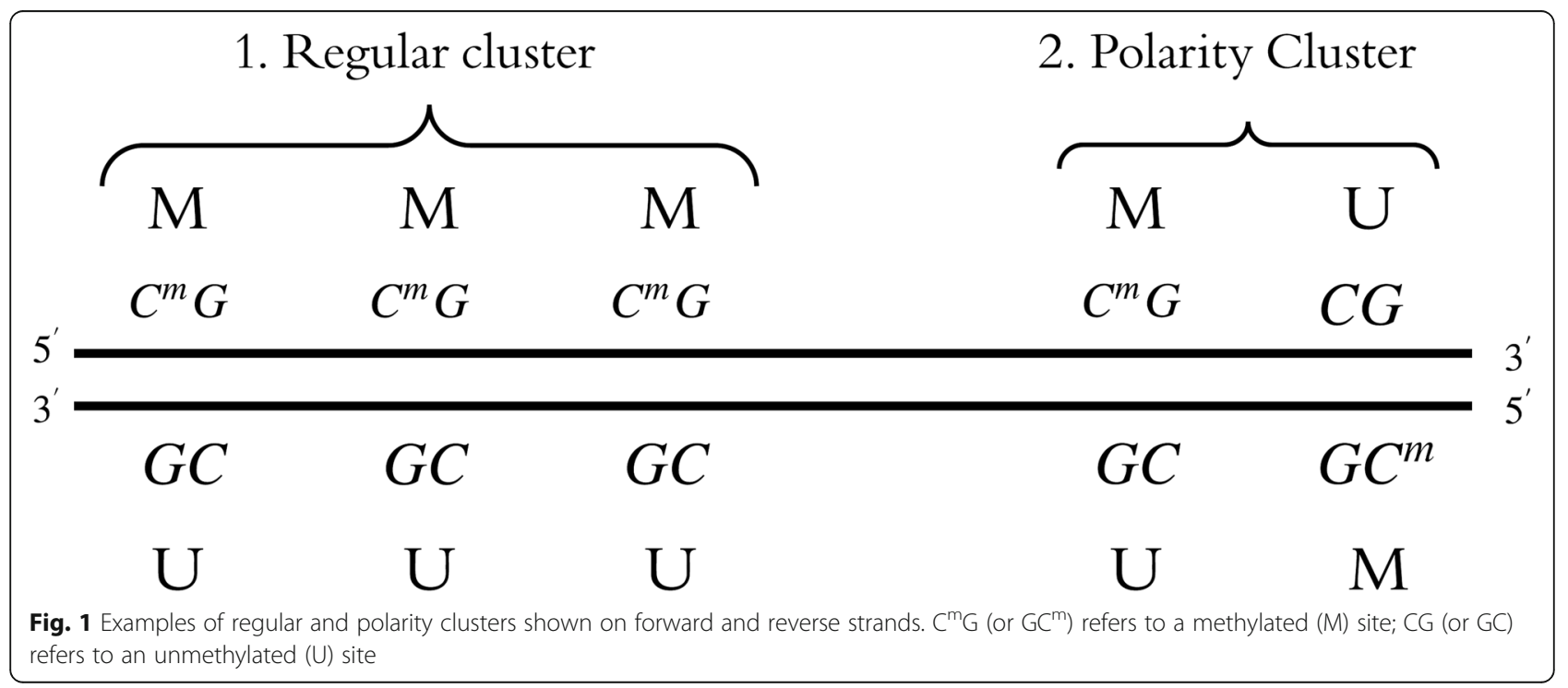


percentages of $\mathrm{CpG}$ sites in regular clusters, polar clusters, and singleton points are summarized. All these summarized results of tumor and normal samples are further compared using statistical tests. For those CpG sites in clusters, DNA strands in the tumor and adjacent normal cells are compared, and overlapping clusters are identified. We'll show the key findings in the Results section.

\section{Results}

Hemimethylated CpG sites for both normal and tumor cells are identified using the Wilcoxon tests. Table 1 describes the proportions of hemimethylation sites that are in clusters based on the $p$-value $(p<0.05)$ and three mean difference cutoff values. There are similar numbers of hemimethylation sites in tumor and normal samples, but the proportion in clusters is slightly higher in normal samples. When comparing the proportions between normal and tumor, we get the following three $p$-values, $0.00039,0.00035$, and 0.277 . These $p$-values correspond to the three mean difference cutoff values $0.4,0.6$, and 0.8 respectively. For the rest of this paper, our analysis will focus on the hemimethylation sites identified based on the $p$-value of 0.05 and the absolute mean difference greater than or equal to 0.4 .

Tumor and normal samples' hemimethylation CpG sites are compared in Table 2. The first row of this table, i.e., the T.MU row, indicates the total number of MU hemimethylation CpG sites in tumor (T) cells. Among these sites, 1697 of them are also hemimethylated in normal cells (N.MU), 1688 of them are not significantly hemimethylated in normal (N.NS), and 217 of them have no data in normal cells (N.NA). The first column of Table 2, i.e., the N.MU column, shows the total number of MU hemimethylation CpG sites in normal (N) cells. Among these sites, 1697 of them are also hemimethylated in tumor cells (T.MU), 1728 of them are not significantly hemimethylated in tumor (T.NS), and 268 of them have no data in tumor cells (T.NA).

Tumor and normal samples' hemimethylation clusters are compared in Table 3. This table shows that most clusters only have two or three CpG sites and cluster frequency decreases with increased cluster length, meaning large congregations of hemimethylation are infrequent. The length of a cluster is defined as the total
Table 2 Comparison of normal and tumorous hemimethylation site patterns

\begin{tabular}{lllll}
\hline & N.MU & N.UM & N.NS & N.NA \\
\hline T.MU & 1697 & 0 & 1688 & 217 \\
T.UM & 0 & 1597 & 1892 & 239 \\
T.NS & 1728 & 1789 & $1,895,429$ & 101,322 \\
T.NA & 268 & 272 & 98,209 & $27,295,013$
\end{tabular}

Each row is for the tumor $(\mathrm{T})$ sample and each column is for the normal $(\mathrm{N})$ sample with various hemimethylation types. T.MU refers to $\mathrm{CpG}$ sites that are methylated $(\mathrm{M})$ on the forward strand and unmethylated $(\mathrm{U})$ on the reverse strand in tumor (T) samples. N.MU refers to $\mathrm{CpG}$ sites with the MU hemimethylation in normal (N) samples. T.NS and N.NS refer to CpG sites of a corresponding tissue type that are not significantly hemimethylated. Similarly, T.NA and N.NA refer to CpG sites that have no data for the given cell type

number of base pairs between the first and the last $\mathrm{CpG}$ sites in the cluster. Figure 2 shows four histograms of cluster lengths. These histograms display the length distributions of polarity patterns in tumor, polarity patterns in normal, regular patterns in tumor, and regular patterns in normal samples. Regular and polarity patterns are analyzed separately because polarity clusters tend to be much shorter. In fact, many of the polarity clusters are less than 40 base pairs long, and a majority of them are less than 10 base pairs long (see peaks in the top panels of Fig. 2). Many of the regular clusters are relatively short, i.e., less than 60 base pairs long, but a small amount of them are longer than that with a maximum length of around 100 to 120 base pairs. A Wilcoxon rank-sum test is performed to compare the difference between the lengths of clusters in normal and tumor cells. The test result is insignificant ( $p$-value $=0.12$ ).

For the two main hemimethylation cluster patterns, regular cluster and polarity cluster, we summarize them in detail in Tables 4 and 5 . Table 4 describes the proportions of different regular clusters in normal and tumor DNA. Table 5 describes the proportions of different polarity patterns in normal and tumor DNA. Polarity clusters appear less frequently than regular patterns, as seen by the difference in the number of sites between Tables 4 and 5. For example, tumor samples have a total of 477 regular clusters and only 36 polar clusters.

One way to detect which clusters may be related to cancer is to compare the cluster locations between tumor DNA and normal DNA. Some clusters may appear in the same sites in both tumor and normal

Table 1 Number of hemimethylated CpG sites and percentage of sites in clusters

\begin{tabular}{|c|c|c|c|c|c|c|}
\hline \multirow{2}{*}{$\begin{array}{l}\text { |Mean } \\
\text { difference| }\end{array}$} & \multicolumn{3}{|c|}{ Normal } & \multicolumn{3}{|c|}{ Tumor } \\
\hline & Total & Sites in clusters & Percentage & Total & Sites in clusters & Percentage \\
\hline$\geq 0.4$ & 7351 & 1510 & $20.54 \%$ & 7330 & 1336 & $18.23 \%$ \\
\hline$\geq 0.6$ & 2588 & 348 & $13.45 \%$ & 2743 & 282 & $10.28 \%$ \\
\hline$\geq 0.8$ & 723 & 53 & $7.33 \%$ & 823 & 49 & $5.95 \%$ \\
\hline
\end{tabular}

Each row is for a mean difference level. The two panels (three columns each) are for normal and tumor samples respectively 
Table 3 Normal and tumor hemimethylation cluster patterns

\begin{tabular}{|c|c|c|}
\hline Cluster Pattern & Normal & Tumor \\
\hline 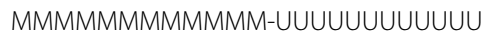 & 1 & 1 \\
\hline мMмммммMмM-UUUUUUUUUU & 1 & 1 \\
\hline MMMMMMMM-UUUUUUUU & 2 & 2 \\
\hline MMMMMMM-UUUUUUU & 2 & 2 \\
\hline MMMMMM-UUUUUU & 5 & 3 \\
\hline MMMMM-UUUUU & 6 & 7 \\
\hline MMMM-UUUU & 18 & 13 \\
\hline MMM-UUU & 55 & 32 \\
\hline MM-UU & 168 & 153 \\
\hline MMU-UUM & 0 & 1 \\
\hline MU-UM & 28 & 32 \\
\hline UMM-MUU & 1 & 0 \\
\hline UM-MU & 7 & 4 \\
\hline UUM-MMU & 1 & 0 \\
\hline UU-MM & 195 & 172 \\
\hline UUU-MMM & 52 & 44 \\
\hline UUUU-MMMM & 22 & 22 \\
\hline UUUUU-MMMMM & 9 & 14 \\
\hline UUUUUU-MMMMMM & 3 & 4 \\
\hline UUUUUUM-MMMMMMU & 0 & 1 \\
\hline UUUUUUU-MMMMMMM & 4 & 3 \\
\hline UUUUUUUM-MMMMMMMU & 1 & 0 \\
\hline UUUUUUUU-MMMMMMMM & 2 & 2 \\
\hline Total & 583 & 513 \\
\hline
\end{tabular}

The first column is the cluster pattern, separating forward and reverse strands by "-". The second and third columns are the counts of such patterns in normal and tumor samples respectively

samples, but others may be found only in tumor or only in normal. In Fig. 3, we show two typical hemimethylation clusters: one that is only identified in tumor DNA and one that is only identified in normal DNA. The first two pairs of bars represent two CpG sites in normal DNA. The second two represent two CpG sites in tumor DNA. We see in the first (or left) plot that there is a large difference between the forward and reverse strands in the tumor CpG sites, whereas the normal CpG sites are quite similar. This tells us that there is a cluster containing two CpG sites that is found only in tumor DNA. Similarly, the second (or right) plot describes a cluster that appears only in normal DNA. In fact, there is almost no methylation in the tumor reverse strands, while the normal reverse strands are almost fully methylated. The forward strand methylation levels are similarly low in tumor and normal DNA, so we observe normal-only hemimethylation in the two sites.

In order to study hemimethylation patterns thoroughly, we compare the 513 tumor clusters with the 583 normal clusters and summarize the results in Table 6 . This table shows that multiple kinds of overlaps can be found between tumor and normal. Hemimethylation clusters that occur only in tumor or normal samples are shown in Column B. 695 (313 tumor only and 382 normal only) clusters fall into these categories, and these are the clusters or regions that may be associated with cancer. Column $\mathrm{C}$ counts the number of clusters that are exactly the same for normal and tumor. Column D indicates the situations in which a tumor cluster begins and ends within a normal cluster (i.e., a tumor cluster contained within the bounds of a normal cluster), and vice versa as shown in Column E. For example, a tumor cluster's start and end positions on a chromosome are 150 and 170 base pairs. It is located within a normal cluster that has the start and end positions of 120 and 190 base pairs. Column D, which represents tumor clusters that are embedded in normal clusters, shows different counts for normal and tumor samples because there are two instances of multiple normal clusters located in one tumor cluster. Similarly, Column E, which represents normal clusters that are embedded in tumor clusters, shows different counts because there are three tumor clusters that are located in one normal cluster. Column F represents all other kinds of overlap. For example, there are two normal clusters that have some overlap with the same tumor cluster.

The tumor data row of Table 6 shows that among the 513 tumor clusters, 313 of them belong to tumor only; 140 clusters also show up in normal samples; 25 tumor clusters are short ones and they are located within long normal clusters; 23 tumor clusters are long ones in which short normal clusters are located; and 12 tumor clusters are partially overlapped with normal clusters. The normal data row of Table 6 shows that among the 583 normal clusters, 382 of them belong to normal only; 140 clusters also show up in tumor samples; 23 normal clusters are long ones and they cover short tumor clusters; 25 normal clusters are short ones and they are located within long tumor clusters; and 13 normal clusters are partially overlapped with tumor clusters. A detailed version of Table 6 is shown in the Supplemental Table 1 of the Additional File 1, in which the number of different clusters in each chromosome is listed for both tumor and normal samples.

After identifying hemimethylated $\mathrm{CpG}$ sites, we may also map them back to genes. That is, we provide the annotation for each CPG site by providing the gene name in whose gene body or promoter region a hemimethylation site is located. We call this analysis gene annotation, and summarizing such will provide the frequency on how many hemimethylated CpG sites a gene has. This annotation analysis is important because highly hemimethylated genes may play an important 
Polarity cluster length in Tumor

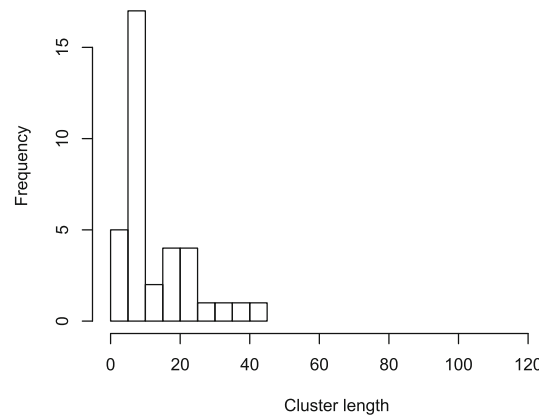

Regular cluster length in Tumor

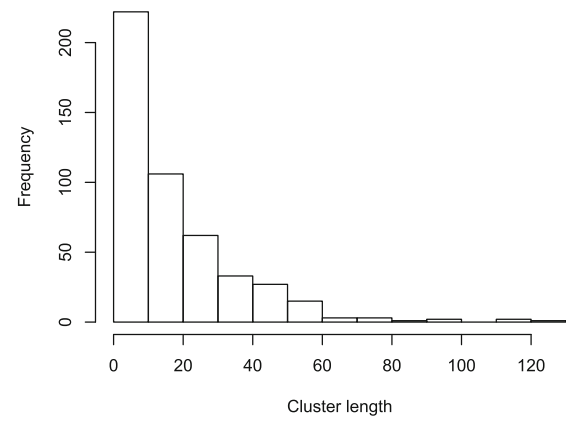

Polarity cluster length in Normal

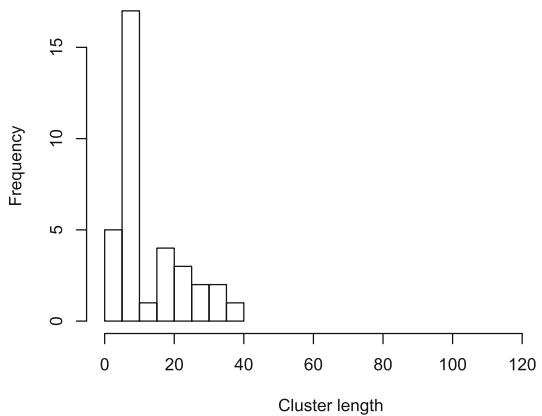

Regular cluster length in Normal

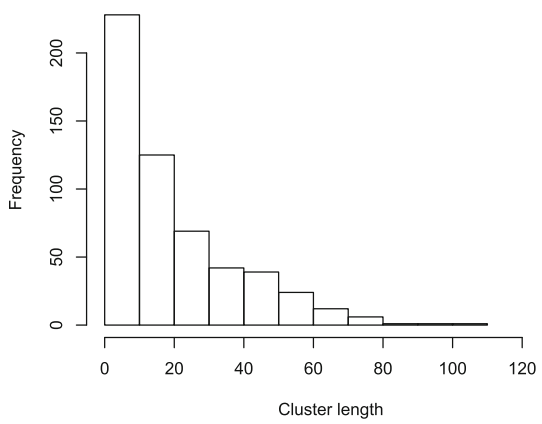

Fig. 2 Length of clusters for both normal and tumor samples

role. Table 7 shows the frequency of hemimethylated CpG sites in gene bodies. Each column shows how many genes have $n$ hemimethylated CpG sites in their gene bodies, where $n$ is given in the first row. The second row describes the distribution for tumor genes and the third row describes the distribution for normal genes. Similarly, Table 8 describes the frequency of hemimethylated CpG sites in promoter regions. Table 7 displays that the large majority of gene bodies have at most three hemimethylated $\mathrm{CpG}$ sites in both tumor and normal samples, but a few have more than 10. When looking at promoter regions, Table 8 shows none have 10 or more and the large majority of genes have one or two hemimethylated CpG sites.

With the gene annotation analysis, we can identify genes that have relatively more hemimethylation sites. In particular, we select the genes that have at least five

Table 4 Regular clusters with corresponding percentages

\begin{tabular}{lllll}
\hline Regular Clusters & Normal & \multicolumn{3}{c}{ Tumor } \\
\hline MM-UU & 168 & $30.66 \%$ & 153 & $32.075 \%$ \\
UU-MM & 195 & $35.58 \%$ & 172 & $36.059 \%$ \\
Bigger cluster & 185 & $33.76 \%$ & 152 & $31.866 \%$ \\
Total & 548 & $100 \%$ & 477 & $100 \%$
\end{tabular}

Bigger clusters (see the fourth row) are the ones with 3 or more hemimethylated $\mathrm{CpG}$ sites hemimethylation sites in tumor only, in normal only, and in both normal and tumor samples. These genes are summarized in Tables 9, 10, and 11 respectively. Note, there are not many genes with a large number of hemimethylated sites. Therefore, we choose a relatively small number (i.e., five) to find a reasonable number of genes that meet this criterion for us to do further analysis. In addition, the datasets used in this project are generalized using the RRBS method. For this method, only a small percent of the CpG sites in a genome are sequenced [12, 16]. If the methylation sequencing datasets used in this study are generated based on the whole genome bisulfite sequencing method, more hemimethylated $\mathrm{CpG}$ sites can be found in different genes.

There are 41 genes with the most hemimethylation in tumor DNA, see Table 9. Among these genes, TP73 [17-19], GNAS [20-24], and NOTCH1 $[25,26]$ are notable ones with known relations to cancer. Table 9 shows that among these 41 genes, one is a tumor suppressor (WT1), three are oncogenes (GNAS, NOTCH1, and

Table 5 Polarity clusters with corresponding percentages

\begin{tabular}{lllll}
\hline Polarity Clusters & Normal & \multicolumn{3}{c}{ Tumor } \\
\hline MU-UM & 28 & $80 \%$ & 32 & $88.89 \%$ \\
UM-MU & 7 & $20 \%$ & 4 & $11.11 \%$ \\
Total & 35 & $100 \%$ & 36 & $100 \%$ \\
\hline
\end{tabular}




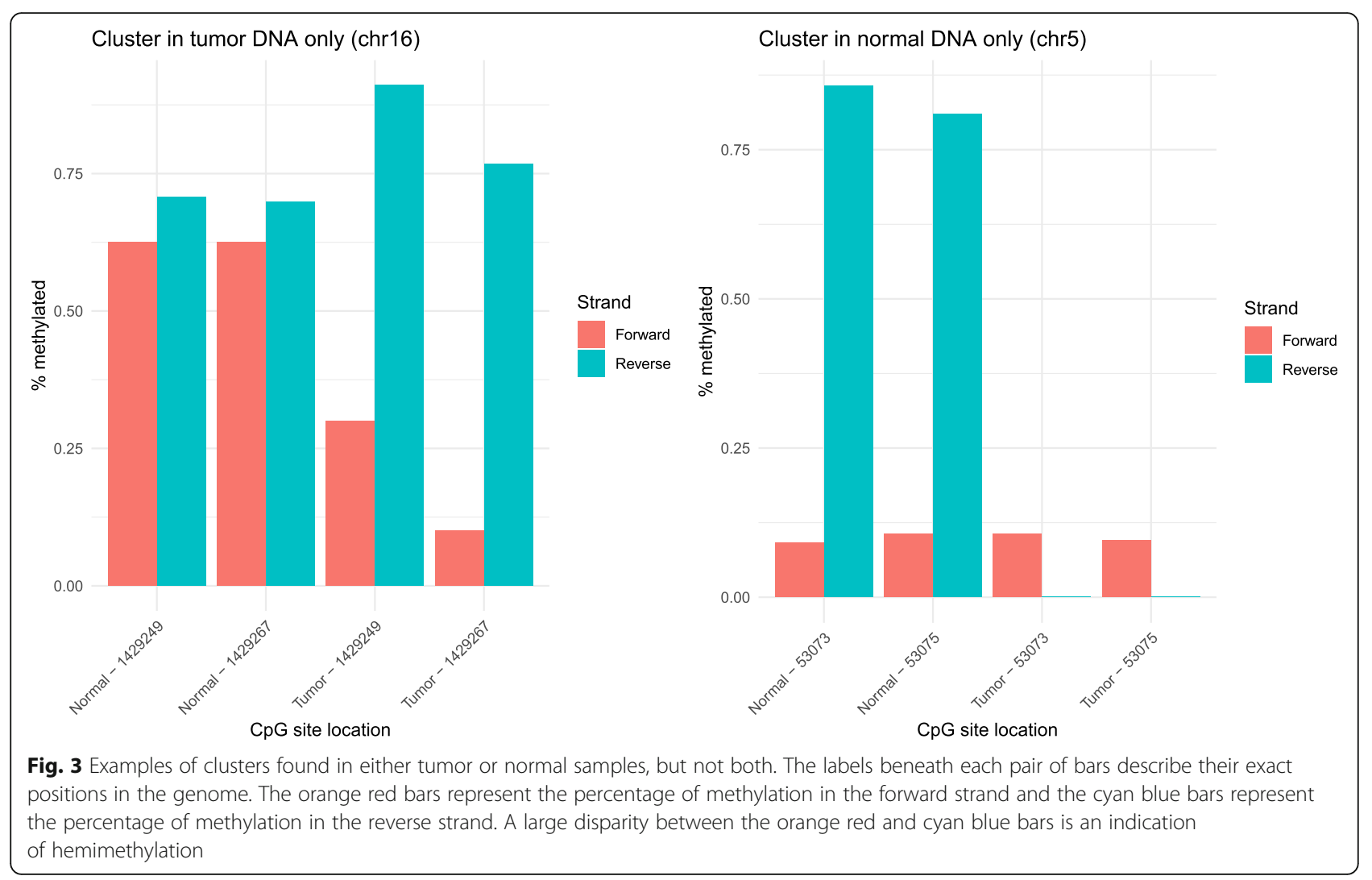

PRDM16), and of those three, two are translocated cancer genes (NOTCH1 and PRDM16). There are also eight transcription factors in this table (HDAC4, IRX2, NFATC1, PRDM16, RUNX3, SIX3, TP73, and WT1). Table 10 shows 35 genes with the most hemimethylation in normal DNA. Among these genes, four are oncogenes (CBFA2T3, GNAS, PDGFB and PRDM16). Of the oncogenes, three are translocated cancer genes (CBFA2T3, PDGFB and PRDM16). There are also seven transcription factors in this table (CBFA2T3, HOXA3, IRX2, MEIS1, NFIC, PRDM16, and ZFPM1). Note that no tumor suppressor genes are hemimethylated in the normal cells. For genes belonging to two key gene families (i.e., transcription factor and oncogene), we have compared their proportions in tumor and normal samples using statistical tests. The test $p$-values are 0.96 for the transcript factor family and 0.54 for the oncogene family. There is no significant difference. Table 11 shows 36 genes with the most hemimethylation in both normal and tumor DNA. Among these genes, two are oncogenes and also translocated cancer genes (CBFA2T3 and PRDM16). There are also six transcription factors in this table (KLF5, HOXA2, CBFA2T3, HOXA3, ISL2, and PRDM16). All three gene tables have some transcription factor genes, which may affect the gene expression of other cancer-related genes that are not found to be hemimethylated.

In order to understand the functions and relationships of these genes, we further analyze their biological interactions using the ConsensusPath Database (CPDB) software package [27-29], see Figs. 4, 5, 6, and 7. Figure 4 describes the different types of biological relationships between genes based on the CPDB software. A gene with a black label is known to be hemimethylated (i.e.,

Table 6 Tumor and normal cluster comparison results

\begin{tabular}{|c|c|c|c|c|c|}
\hline $\mathrm{A}$ & B & $C$ & D & $E$ & $F$ \\
\hline Tumor Total & Tumor Only & Exact Overlap & Tumor in Normal & Normal in Tumor & Other Overlap \\
\hline 513 & 313 & 140 & 25 & 23 & 12 \\
\hline Normal Total & Normal Only & Exact Overlap & Tumor in Normal & Normal in Tumor & Other Overlap \\
\hline 583 & 382 & 140 & 23 & 25 & 13 \\
\hline
\end{tabular}

Columns are for different overlap (or non-overlap) patterns. The two rows are for tumor and normal, respectively 
Table 7 Hemimethylation frequency measured in gene bodies for both tumor and normal samples

\begin{tabular}{|c|c|c|c|c|c|c|c|c|c|c|}
\hline No. of Hemimethylation sites per gene body $(n)$ & 1 & 2 & 3 & 4 & 5 & 6 & 7 & 8 & 9 & $\geq 10$ \\
\hline Tumor & 1133 & 250 & 79 & 37 & 17 & 4 & 7 & 2 & 0 & 4 \\
\hline Normal & 1118 & 229 & 73 & 32 & 11 & 4 & 3 & 1 & 1 & 5 \\
\hline
\end{tabular}

identified by our analysis). A gene with a purple label is not provided in our hemimethylation gene list but interacts with one of the known genes. Figure 4 is the legend for Figs. 5, 6, and 7. This legend figure summarizes the relationships for gene lists in Tables 9, 10, and 11 as shown in Figs. 5, 6, and 7, respectively. These figures show the extent to which these highly hemimethylated genes interact and possibly affect the cell function of related genes.

Figure 5 shows genetic interactions between genes with the most hemimethylation in tumor samples, and these genes are recorded in Table 9. The gene network in Fig. 5 contains a number of hub genes with complex interactions. These hub genes include GNAS, NFATC1, NOTCH1, MAPK1, HOAC4, TP73, and EGR1. We can see that if a hub gene like MAPK1 is hemimethylated, it may interact with dozens of other genes. Some of these genes, e.g., EGR1 [30-33] and UNC5B [34-37], are known to be associated with different cancers, including lung cancer. EGR1 has a promoting effect on cancer metastasis in OCT4-overexpressing lung cancer [38]. The pseudogene DUXAP8 may act as an oncogene in nonsmall cell lung cancer, and it may play this role by silencing EGR1 and RHOB transcription via binding with EZH2 and LSD1 [39]. The expression of UNC5A, $\mathrm{UNC5B}$, or UNC5C is down-regulated in multiple cancers including lung cancer [40], and UNC5B has also been indicated as a putative tumor suppressor [41].

Figure 6 shows genetic interactions between genes with the most hemimethylation in normal DNA, and these genes are recorded in Table 10. In this figure, we can see that GNAS is a hub gene interacting with many other genes that may not be hemimethylated themselves. GNAS is observed in both tumor and normal samples, as well as in the hemimethylation study for breast cancer cell lines [9]. MEIS1 is also a hub gene that interacts with genes like KMT2A [42] and TK1 [43]. While these genes are not hemimethylated in our samples, they are known to be associated with cancer. KMT2A and hTERT are positively correlated in melanoma tumor tissues, and KMT2A promotes melanoma cell growth by targeting the hTERT signaling pathway [44]. KMT2A has an epigenetic regulation role on $\mathrm{NOTCH} 1$ and $\mathrm{NOTCH} 3$, and this mechanism is essential for inhibiting glioma proliferation [45]. TK1 plays a moderate role as a diagnostic tumor marker for cancer patients [46], and it is a potential clinical biomarker for the treatment of lung, breast, and colorectal cancer [47]. A systematic review shows that TK1 overexpression is associated with the poor outcomes of lung cancer patients [48]. MEIS1 inhibits non-small cell lung cancer cell proliferation [49]. MEIS1 plays a crucial role in normal development [15] and it is also reported as an important gene related to leukemia [50-52]. Therefore, it is possible that the hemimethylation of hub genes like MEIS1 affects protein, biochemical, or regulatory functions of genes that are associated with cancer.

Figure 7 shows genetic interactions between genes with the most hemimethylation on identical locations in tumor and normal samples. These genes are recorded in Table 11. This means that the hemimethylation of $\mathrm{CpG}$ sites in this network is unchanged or unaffected by the formation of cancer. The HNRNPL gene is a major hub in this gene network. While we do not detect any hemimethylation in this gene, it directly interacts with 10 genes that we know to be hemimethylated. Some of these genes, like PTPRN2 and MAD1L1, can also be found in the tumor gene network, see Fig. 5. There appears to be no common genes between Fig. 6 (hemimethylated genes in normal samples) and Fig. 7 (hemimethylated genes in both tumor and normal samples). Therefore, genes that have a large number of hemimethylated $\mathrm{CpG}$ sites found only in normal DNA seem to have few $\mathrm{CpG}$ sites that remain the same when cancer forms.

In addition to the above analysis, we have conducted gene set enrichment analysis using the molecular signature database and the related software package provided by the Broad Institute [14]. Of the most hemimethylated genes in tumor DNA (Table 9), six are also significantly represented in cancer module 163 (with $p$-value $<0.05$ ). This module is a collection of genes known to be

Table 8 Hemimethylation frequency measured in promoter regions for both tumor and normal samples

\begin{tabular}{|c|c|c|c|c|c|c|c|c|}
\hline No. of Hemimethylation sites per promoter region $(n)$ & 1 & 2 & 3 & 4 & 5 & 6 & 7 & 8 \\
\hline Tumor & 223 & 23 & 5 & 6 & 0 & 2 & 0 & 1 \\
\hline Normal & 256 & 36 & 13 & 3 & 2 & 1 & 1 & 0 \\
\hline
\end{tabular}


Table 9 For genes with at least five hemimethylation sites in tumor samples

\begin{tabular}{|c|c|c|c|}
\hline Gene name & Count & Family & Gene Description \\
\hline RGS14 & 17 & - & regulator of $\mathrm{G}$ protein signaling 14 \\
\hline MEX3A & 16 & - & mex-3 RNA binding family member A \\
\hline WT1 & 11 & TF, TS & WT1 transcription factor \\
\hline PRDM16 & 10 & OG, TF, TCG & PR/SET domain 16 \\
\hline ZDHHC9 & 10 & - & zinc finger DHHC-type containing 9 \\
\hline AGAP2 & 8 & - & ArfGAP with GTPase domain, ankyrin repeat and PH domain 2 \\
\hline GNAS & 8 & OG & GNAS complex locus \\
\hline EXOC3L2 & 8 & - & exocyst complex component 3 like 2 \\
\hline PTPRN2 & 7 & - & protein tyrosine phosphatase receptor type N2 \\
\hline FANK1 & 7 & - & fibronectin type III and ankyrin repeat domains 1 \\
\hline UNC93B1 & 7 & - & unc-93 homolog B1, TLR signaling regulator \\
\hline IGSF9B & 7 & - & immunoglobulin superfamily member $9 B$ \\
\hline GNAS-AS1 & 7 & - & GNAS antisense RNA 1 \\
\hline MAD1L1 & 7 & - & mitotic arrest deficient 1 like 1 \\
\hline TSPAN9 & 7 & - & tetraspanin 9 \\
\hline PTPRM & 7 & - & protein tyrosine phosphatase receptor type M \\
\hline TP73 & 6 & TF & tumor protein p73 \\
\hline IFT140 & 6 & - & intraflagellar transport 140 \\
\hline NFATC1 & 6 & TF & nuclear factor of activated T cells 1 \\
\hline DGKA & 6 & - & diacylglycerol kinase alpha \\
\hline FMNL1 & 6 & - & formin like 1 \\
\hline CACNA1I & 6 & - & calcium voltage-gated channel subunit alpha1 I \\
\hline LOC101927636 & 6 & - & RNA Gene affiliated with the IncRNA class \\
\hline HDAC4 & 5 & TF & histone deacetylase 4 \\
\hline $\mathrm{IRX} 2$ & 5 & $\mathrm{TF}, \mathrm{HP}$ & iroquois homeobox 2 \\
\hline ANKRD33B & 5 & - & ankyrin repeat domain 33B \\
\hline LINC00537 & 5 & - & Long Intergenic Non-Protein Coding RNA 537 \\
\hline $\mathrm{NOTCH} 1$ & 5 & OG, TCG & notch receptor 1 \\
\hline ANO2 & 5 & - & anoctamin 2 \\
\hline CACNA1H & 5 & - & calcium voltage-gated channel subunit alpha1 $\mathrm{H}$ \\
\hline RUNX3 & 5 & TF & runt related transcription factor 3 \\
\hline SIX3 & 5 & $\mathrm{TF}, \mathrm{HP}$ & SIX homeobox 3 \\
\hline FZD7 & 5 & - & frizzled class receptor 7 \\
\hline ADGRA2 & 5 & - & adhesion $\mathrm{G}$ protein-coupled receptor A2 \\
\hline IFFO1 & 5 & - & intermediate filament family orphan 1 \\
\hline CHTF18 & 5 & - & chromosome transmission fidelity factor 18 \\
\hline TMEM204 & 5 & - & transmembrane protein 204 \\
\hline RECQL5 & 5 & - & RecQ like helicase 5 \\
\hline SMIM5 & 5 & - & small integral membrane protein 5 \\
\hline MAPK1 & 5 & PK & mitogen-activated protein kinase 1 \\
\hline SYN1 & 5 & - & synapsin I \\
\hline
\end{tabular}

The gene name, corresponding number of hemimethylated sites (i.e., count), specified gene family, and a description of the gene are formatted in the table's respective columns. Descriptions are derived from the Molecular Signature Database [14] and the GeneCards database [15]. Certain genes are indicated as members of specific gene families, as shown in the third column: "TF" for transcription factor, "TS" for tumor suppressor, "OG" for oncogene, "HP" for homeodomain protein, "TCG" for translocated cancer gene, and "PK" for protein kinase 
Table 10 For genes with at least five hemimethylation sites in normal samples

\begin{tabular}{|c|c|c|c|}
\hline Gene name & Count & Family & Gene Description \\
\hline$\overline{Z F P M 1}$ & 14 & TF & zinc finger protein, FOG family member 1 \\
\hline GNAS & 13 & OG & GNAS complex locus \\
\hline RGPD2 & 12 & - & RANBP2 like and GRIP domain containing 2 \\
\hline SHANK3 & 11 & - & SH3 and multiple ankyrin repeat domains 3 \\
\hline IRX2 & 10 & $\mathrm{TF}, \mathrm{HP}$ & iroquois homeobox 2 \\
\hline LTB4R & 9 & - & leukotriene B4 receptor \\
\hline CPEB3 & 8 & - & cytoplasmic polyadenylation element binding protein 3 \\
\hline PTPRN2 & 7 & - & protein tyrosine phosphatase receptor type N2 \\
\hline MIR1268A & 7 & - & microRNA 1268a \\
\hline GNAS-AS1 & 7 & - & GNAS antisense RNA 1 \\
\hline CYP26C1 & 7 & - & cytochrome P450 family 26 subfamily C member 1 \\
\hline TBL1XR1 & 6 & - & transducin beta like $1 \mathrm{X}$-linked receptor 1 \\
\hline HOXA3 & 6 & $\mathrm{TF}, \mathrm{HP}$ & homeobox A3 \\
\hline CACNA1H & 6 & - & calcium voltage-gated channel subunit alpha1 $\mathrm{H}$ \\
\hline NPEPPS & 6 & - & aminopeptidase puromycin sensitive \\
\hline SEMA6B & 6 & CGF & semaphorin 6B \\
\hline HOMER3 & 6 & - & homer scaffold protein 3 \\
\hline PINLYP & 6 & - & phospholipase A2 inhibitor and LY6/PLAUR domain containing \\
\hline GDI1 & 6 & - & GDP dissociation inhibitor 1 \\
\hline HS3ST2 & 6 & - & heparan sulfate-glucosamine 3-sulfotransferase 2 \\
\hline PRDM16 & 5 & TF, OG, TCG & PR/SET domain 16 \\
\hline PLK3 & 5 & PK & polo like kinase 3 \\
\hline GREM2 & 5 & CGF & gremlin 2, DAN family BMP antagonist \\
\hline MEIS1 & 5 & $\mathrm{TF}, \mathrm{HP}$ & Meis homeobox 1 \\
\hline MEIS1-AS2 & 5 & - & MEIS1 antisense RNA 2 \\
\hline POLH & 5 & - & DNA polymerase eta \\
\hline HOXA-AS2 & 5 & - & HOXA cluster antisense RNA 2 \\
\hline EBF3 & 5 & - & EBF transcription factor 3 \\
\hline CBFA2T3 & 5 & TF, OG, TCG & CBFA2/RUNX1 translocation partner 3 \\
\hline RPL13 & 5 & - & ribosomal protein $\mathrm{L} 13$ \\
\hline NFIC & 5 & TF & nuclear factor I C \\
\hline $\mathrm{CDH} 4$ & 5 & - & cadherin 4 \\
\hline PDGFB & 5 & OG, TCG & cytokine or growth factor, platelet derived growth factor subunit B \\
\hline CCNT1 & 5 & - & cyclin $\mathrm{T} 1$ \\
\hline SNORD68 & 5 & - & small nucleolar RNA, C/D box 68 \\
\hline
\end{tabular}

The gene name, corresponding number of hemimethylated sites (i.e., count), specified gene family, and a description of the gene are formatted in the table's respective columns. Descriptions are derived from the Molecular Signature Database [14] and the GeneCards database [15]. Certain genes are indicated as members of specific gene families, as shown in the third column: "TF" for transcription factor, "TS" for tumor suppressor, "OG" for oncogene, "HP" for homeodomain protein, "TCG" for translocated cancer gene, and "PK" for protein kinase

overrepresented in cancer pathways and is reported by the Stanford research group (http://robotics.stanford. edu/ erans/cancer/modules/). The six genes are IFT140, IFFO1, SYN1, FMNL1, NOTCH1, and RGS14. There are no such overly represented genes and cancer modules among genes shown in Table 10 (for normal samples) and Table 11 (for both tumor and normal samples).

\section{Discussion}

It was previously believed that hemimethylation appears only in a transient state [4]. However, Shao et al. have reported hemimethylated sites and patterns in ovarian cancer [7]. Sun et al. have identified hemimethylation patterns in breast cancer cell lines [9]. Furthermore, Xu and Corces have shown that some hemimethylation sites 
Table 11 For genes with at least five hemimethylation sites in both tumor and normal samples

\begin{tabular}{|c|c|c|c|}
\hline Gene name & Count & Family & Gene Description \\
\hline RGPD5 & 16 & - & RANBP2 like and GRIP domain containing 5 \\
\hline RGPD8 & 16 & - & RANBP2 like and GRIP domain containing 8 \\
\hline ROCK1P1 & 13 & - & Rho associated coiled-coil containing protein kinase 1 pseudogene 1 \\
\hline THAP4 & 8 & - & THAP domain containing 4 \\
\hline SGTA & 8 & - & small glutamine rich tetratricopeptide repeat containing alpha \\
\hline PTPRN2 & 7 & - & protein tyrosine phosphatase receptor type N2 \\
\hline CNTNAP3 & 7 & - & contactin associated protein like 3 \\
\hline NUTM2A-AS1 & 7 & - & NUTM2A antisense RNA 1 \\
\hline RBFOX3 & 7 & - & RNA binding fox-1 homolog 3 \\
\hline ESPNP & 6 & - & espin pseudogene \\
\hline FOXK1 & 6 & - & forkhead box K1 \\
\hline HOXA3 & 6 & HP, TF & homeobox A3 \\
\hline LMF1 & 6 & - & lipase maturation factor 1 \\
\hline USP45 & 6 & - & ubiquitin specific peptidase 45 \\
\hline LOC101928782 & 6 & - & RNA Gene affiliated with the IncRNA class \\
\hline PRDM16 & 5 & OG, TF, TCG & PR/SET domain 16 \\
\hline RGPD4 & 5 & - & RANBP2 like and GRIP domain containing 4 \\
\hline MERTK & 5 & PK & MER proto-oncogene, tyrosine kinase \\
\hline FAM160A1 & 5 & - & family with sequence similarity 160 member A1 \\
\hline PRKAR1B & 5 & - & protein kinase CAMP-dependent type I regulatory subunit beta \\
\hline MAD1L1 & 5 & - & mitotic arrest deficient 1 like 1 \\
\hline HOXA2 & 5 & HP, TF & homeobox A2 \\
\hline DPP6 & 5 & - & dipeptidyl peptidase like 6 \\
\hline $\mathrm{DIP} 2 \mathrm{C}$ & 5 & - & disco interacting protein 2 homolog $C$ \\
\hline FANK1 & 5 & - & fibronectin type III and ankyrin repeat domains 1 \\
\hline GAL3ST3 & 5 & - & galactose-3-O-sulfotransferase 3 \\
\hline FLJ12825 & 5 & - & RNA Gene affiliated with the IncRNA class \\
\hline KLF5 & 5 & TF & Kruppel like factor 5 \\
\hline ISL2 & 5 & HP, TF & ISL LIM homeobox 2 \\
\hline CBFA2T3 & 5 & OG, TF, TCG & CBFA2/RUNX1 translocation partner 3 \\
\hline SBNO2 & 5 & - & strawberry notch homolog 2 \\
\hline GIPR & 5 & - & gastric inhibitory polypeptide receptor \\
\hline SCAF1 & 5 & - & SR-related CTD associated factor 1 \\
\hline COL6A1 & 5 & - & collagen type $\mathrm{VI}$ alpha 1 chain \\
\hline NEXMIF & 5 & - & neurite extension and migration factor \\
\hline GK5 & 5 & - & glycerol kinase 5 \\
\hline
\end{tabular}

The gene name, corresponding number of hemimethylated sites (i.e., count), specified gene family, and a description of the gene are formatted in the table's respective columns. Descriptions are derived from the Molecular Signature Database [14] and the GeneCards database [15]. Certain genes are indicated as members of specific gene families, as shown in the third column: "TF" for transcription factor, "TS" for tumor suppressor, "OG" for oncogene, "HP" for homeodomain protein, "TCG" for translocated cancer gene, and "PK" for protein kinase

can be inherited across cell divisions. They have also shown that hemimethylated CpG sites account for 4$20 \%$ of the DNA methylome in different cell types [53]. Therefore, hemimethylation may serve as a stable epigenetic mark. In addition, recent papers show that hemimethylation is a characteristic of secondary differential methylation regions that are associated with imprinted genes [54-56]. That is, hemimethylation is a novel epigenetic modification functional for genomic imprinting. All these recent findings challenge the previous prevailing view of hemimethylation. It is unlikely that all hemimethylation sites in a genome are transient; 


\begin{tabular}{|c|c|c|}
\hline Physical entity color & Interaction color & \\
\hline gene & \multirow{6}{*}{$\begin{array}{ll} & \text { protein interaction } \\
& \text { genetic interaction } \\
& \text { biochemical reaction } \\
& \text { gene regulatory interaction } \\
& \text { drug-target interaction }\end{array}$} & Node label color \\
\hline protein & & \multirow{5}{*}{$\begin{array}{l}\text { black node labels denote } \\
\text { seed nodes; } \\
\text { magenta node labels denote } \\
\text { intermediate nodes }\end{array}$} \\
\hline protein complex & & \\
\hline RNA & & \\
\hline compound & & \\
\hline family / unknown & & \\
\hline
\end{tabular}

instead, certain hemimethylation sites or patterns may have a stable and important impact on the overall methylation.

DNA methylation is closely related to other genetic events or patterns, e.g., mutation. There is a significantly positive correlation between differential methylation and tumor mutation burden (i.e., the frequency of certain mutations) as shown in a recent non-small cell lung cancer study [57]. Differential methylation sites are also identified between T53 mutated and T53 wild type tumors [58]. In addition, we have compared our hemimethylated genes in Tables 9 and 10 with mutated genes obtained from publicly available databases. When comparing with mutated cancer driver genes from the Integrative Onco Genomics [59], we find some of these genes in our Tables 9 and 10. In particular, four tumoronly genes from our Table 9 (NOTCH1, GNAS, MAPK1, WT1) and five normal-only genes from our Table 10 (GNAS, TBL1XR1, CPEB3, NPEPPS, CBFA2T3) are in this cancer driver gene list. Thus, about $10 \%$ of our hemimethylated genes are also mutated cancer driver genes. When comparing with the lung cancer mutation genes obtained from the database DriverDBv3 [60], we find that 13 tumor-only genes (in Table 9) and 10 normal-only genes (in Table 10) are in this gene list. That is, about $1 / 3$ of our top

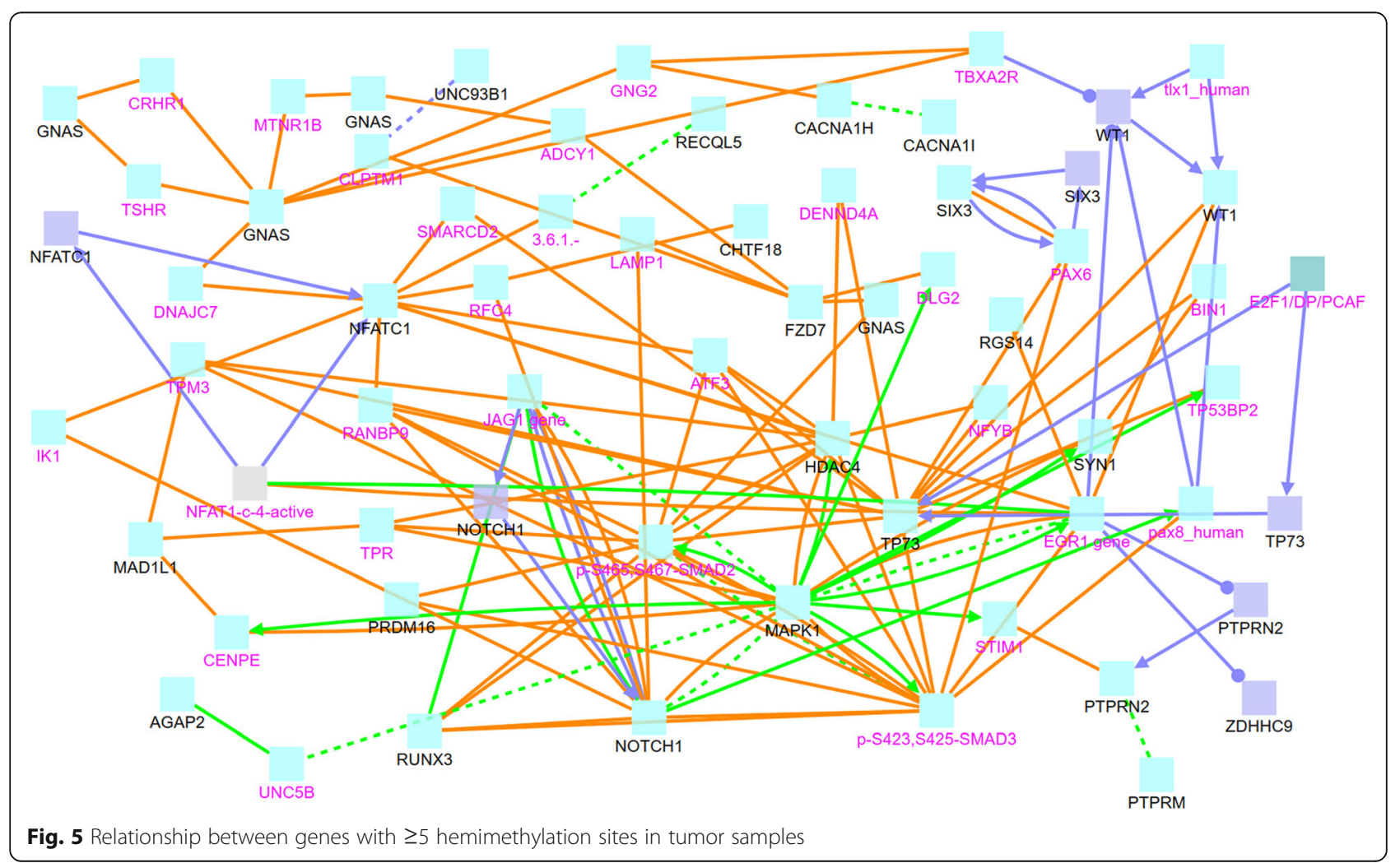




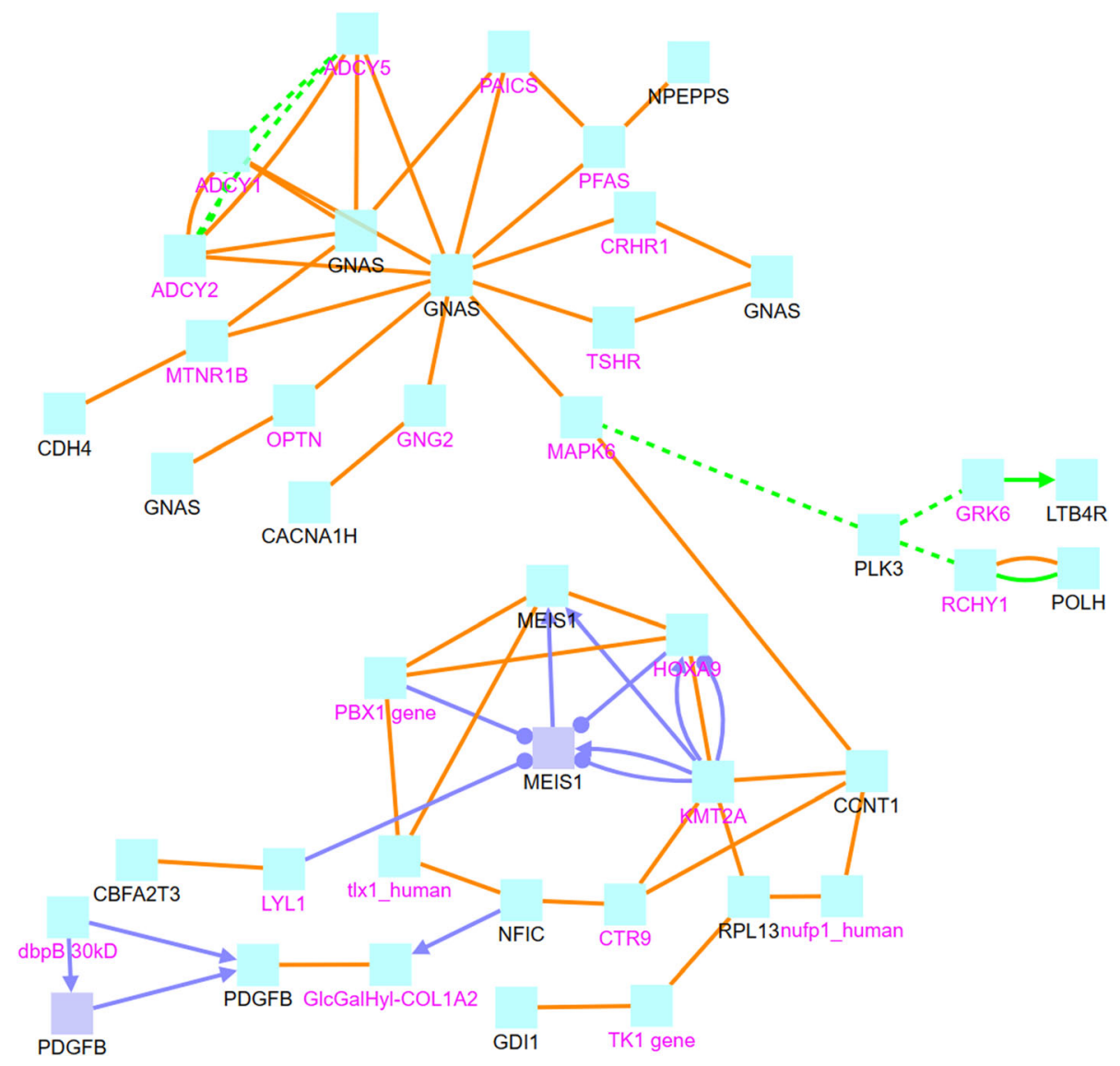

Fig. 6 Relationship between genes with $\geq 5$ Hemimethylation sites in normal samples

hemimethylated genes are lung cancer mutation genes. Note, the 13 tumor-only genes from Table 9 are FMNL1, RGS14, WT1, IFT140, CACNA1H, AGAP2, CACNA1I, ADGRA2, SYN1, GNAS, NFATC1, PRDM16, and MAD1L1. The 10 normal-only genes from Table 10 are CACNA1H, RGPD2, SEMA6B, CYP26C1, GNAS, EBF3, PRDM16, CDH4, MEIS1, and TBL1XR1. The above findings show that methylation and mutation are closely related. It is likely that hemimethylation and mutation are associated as well.

The mean difference cutoff values are 0.4, 0.6 and 0.8 as used in a previous research [9]. Results are narrowed down to the 0.4 cutoff level to allow more results to be viewed, as the higher cutoff values restricted the available hemimethylated $\mathrm{CpG}$ sites from being identified. The number of both tumor and normal clusters detected decreases rapidly as we increase the mean cutoff value at each CpG site as shown in Table 2. With more strict criteria, the methylation difference between the two DNA strands at each CpG site must exist in order for us to consider hemimethylation at a $\mathrm{CpG}$ site. This rapid decrease may indicate certain hemimethylation heterogeneity in lung cancer as cancer methylation patterns are generally heterogeneous among multiple patients or cell lines [61].

For the 41 most hemimethylated genes in lung cancer tumors, seven of them are also highly hemimethylated in breast cancer cell lines, as reported by Sun et al. [9]. These seven genes are PRDM16, GNAS, PTPRN2, MAD1L1, HDAC4, NOTCH1, and CACNA1H. The remaining 34 highly hemimethylated genes in the lung tumor sample are not highly hemimethylated in breast cancer cell lines. It is possible that these genes are unique to lung cancer; thus, it would be helpful when diagnosing patients with lung cancer specifically, but further research needs to be done.

Based upon the outcome of hemimethylation research in breast cancer cell lines, the frequency of polarity clusters is much higher than the one in this paper. The results of breast cancer hemimethylation analysis indicate polarity clusters are more frequently found than regular clusters [9]. However, the lung cancer analysis reflects contrasting results; polarity clusters are less frequently found than regular clusters for both tumor and normal 


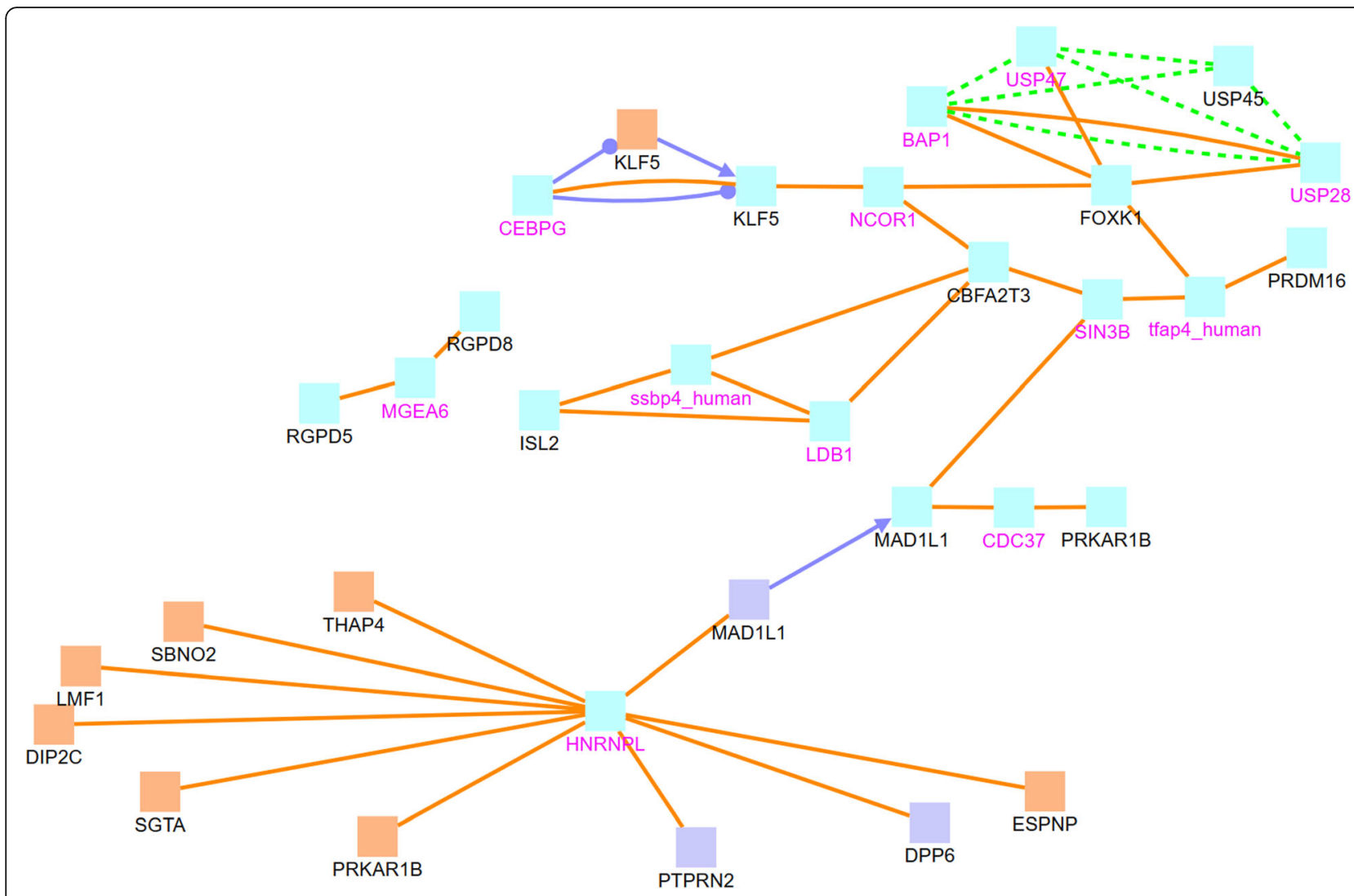

Fig. 7 Relationship between genes with $\geq 5$ hemimethylation sites identical in both tumor and normal samples

samples, as shown in Fig. 2, Tables 4 and 5. A couple of factors may explain this difference. One factor could be the type of cancer, as hemimethylation frequency may be tissue specific. Another factor could be that the previous breast cancer study is performed using cell lines, which are tumors grown in labs over a long period of time; whereas, our current study uses primary tissues directly from lung cancer patients. Due to the nature of cell lines and our primary tissues, it is likely that hemimethylation patterns, especially polarity clusters, are related to tumor growth. Polarity clusters are evidence of active demethylation in cancer cells; DNA demethylation is closely related to cancer hypomethylation [7, 62]. Therefore, the identification of polarity clusters in cancer is of direct importance to the study of carcinogenesis. Future research on the pathological significance of polarity clusters in different tumors may reveal more insight into cancer studies.

After conducting statistical tests for a large number of CpG sites, selecting the significant CpG sites is a crucial step, and the multiple testing correction is important because using only the raw $p$-values may result in many false positive sites. However, for the understudied hemimethylation pattern, the proper way of doing multiple testing correction is not clear. In order to explore the impact of different corrections, we have used three methods: a simple moving-average based method, the comb-p FDR method, and the comb-p SLK method. Note, comb-p is a software package developed for combining, analyzing, and correcting spatially correlated $p$ values [63]. FDR stands for the Benjamini-Hochberg false discovery correction [64]. SLK represents the Stouffer-Liptak-Kechris correction [65]. After exploring various correction methods, we conclude that the mean difference plus $p$-value filtering method used in our study can produce meaningful and interpretable results when dealing with the multiple testing comparison problem for our hemimethylation analysis. For detailed comparative analysis results, see the Supplemental Tables 2, 3 , and 4 and related explanation in the Additional File 1.

As for the criteria we used to identify hemimethylation sites, in addition to $p$-values and mean differences, we may add an additional one. That is, the methylation signal on one DNA strand is zero, and the methylation signal on the other strand is positive. Adding this criteria will help us to identify hemimethylation sites more strictly with no methylation on one strand but a high methylation signal on another strand. This criterion is ideal when the datasets are not very noisy and when the samples are not heterogeneous. In this project, we 
choose to only use $p$-value and mean difference for the following reasons. First, we use these two criteria to make a fair comparison between the previous breast cancer results [9] and our new lung cancer results. Second, the average methylation signals at most CpG sites tend to be clustered around 0 or 1 [66]. Third, bisulfite converted methylation sequencing data can be noisy, and tumor samples' methylation signals are very heterogeneous. We generally consider that the average methylation signals around 0 to 0.2 (or 0.25 ) are still roughly in the category of no or very low methylation signals [61]. Therefore, most of the CpG sites we identified still tend to have relatively high methylation signals on one DNA strand and have relatively low or no methylation signals on another strand.

\section{Conclusion}

Hemimethylation is an important but understudied pattern in cancer. In this paper, we have conducted the first-ever exploratory investigation of hemimethylation in lung cancer. In particular, we have conducted statistical analyses to identify hemimethylation patterns for non-small cell lung cancer patients. We have identified both singleton hemimethylation sites and different clusters in normal and tumor cells. We have also conducted bioinformatic analysis on the genes that have relatively more hemimethylated sites in tumor, normal, and both tumor and normal cells to see the biological interactions of these genes. Our results show that not only does hemimethylation exist in lung cells, but also with diverse patterns and frequencies that are comparable between normal and tumorous cells. We conclude that hemimethylation is related to both normal and tumor cell development. This is also seen by its existence in the same genes in normal and lung tumor cells. However, there are certain genes that only have hemimethylated sites for one type of cell, normal or tumor, but not both. Certain genes are previously known to be associated with carcinogenesis. These genes exhibit existence in one cell type and not the other. Hemimethylation existing in this way may imply epigenetic changes in certain genes associated with lung cancer. The development and progression of lung cancer may be tracked by the analysis of epigenetic change (i.e., hemimethylation and methylation) in these regions.

\footnotetext{
Abbreviations

CpG: The shorthand notation for 5'-cytosine-phosphate-guanine-3'; MU: When it is for one CPG site on two DNA strands, it refers to a hemimethylated $\mathrm{CpG}$ site with methylation (M) on the positive strand and unmethylation $(U)$ on the reverse strand. When it refers to two consecutive CpG sites on one DNA strand, it means that methylation occurs on the first CpG site (i.e., M), but not on the second one (i.e., U); UM: When it is for one CpG site on two DNA strands, it refers to a hemimethylated CpG site with unmethylation $(U)$ on the positive strand and methylation $(M)$ on the reverse strand. When it refers to two consecutive CpG sites on one DNA strand, it means that methylation does not occur on the first CpG site (i.e., U), but
}

occurs on the second one (i.e., M); NS: A CpG site identified as not significantly (NS) hemimethylated; RRBS: Reduced representation bisulfite sequencing

\section{Supplementary Information}

The online version contains supplementary material available at https://doi. org/10.1186/s12885-021-07990-7.

Additional file 1. This file includes two sections. Section 1: Tumor and normal cluster comparison results by chromosome (Supplemental Table 1). Section 2: Comparative analysis of three methods of multiple testing corrections (Supplemental Tables 2-4)

\section{Acknowledgements}

We appreciate the help and support of the Writing Center and the IT colleague of the High Performance Computing Cluster at Texas State University. We are grateful for the four reviewers' thoughtful comments.

\section{Authors' contributions}

SS initiated the project, suggested all key original ideas, and led the whole process. AZ contributed to most of the statistical analysis and the revision of this paper. CF helped with the data analysis and the paper revision. JP did minor coding work in the beginning. AZ and CF contributed significantly to the writing and editing of the manuscript. SS gave suggestions over the course of the project and extensively reviewed and revised the final paper. All authors contributed expertise and edits. All authors have read and approved the final manuscript.

\section{Funding}

This research was partially supported by the NSF-REU grant DMS-1757233 (June - August, 2019). This project is also supported by the Texas State University Research Enhancement Program (an internal award for Dr. Sun). The funders did not play any role in the study design, data analysis, interpretation of data, writing the manuscript, or decision to publish.

\section{Availability of data and materials}

Datasets analyzed for this study are publicly available (SRP125064) and can be downloaded from this web page: https://www.ncbi.nlm.nih.gov/sra/ SRP125064. All datasets supporting our findings are presented within the manuscript and the additional supporting file.

\section{Declarations}

Ethics approval and consent to participate

No ethics approval is required for the study. No permission is required to access the data used in this study because we analyze publicly available datasets.

\section{Consent for publication}

Not applicable.

\section{Competing interests}

The authors declare that they have no competing interests.

\section{Author details \\ ${ }^{1}$ Department of Mathematics, Texas State University, San Marcos, TX, USA 2Department of Statistics, Texas A\&M University, College Station, TX, USA. ${ }^{3}$ Department of Mathematics, Schreiner University, Kerrville, TX, USA. ${ }^{4}$ Department of Mathematics, Applied Mathematics, and Statistics, Case Western Reserve University, Cleveland, $\mathrm{OH}$, USA.}

Received: 16 March 2020 Accepted: 1 March 2021

Published online: 12 March 2021

\section{References}

1. American Cancer Society (www.cancer.org). Accessed 21 Feb 2021.

2. Howlader N, Noone AM, Krapcho M, Miller D, Brest A, Yu M, Ruhl J, Tatalovich Z, Mariotto A, Lewis DR, et al. SEER Cancer Statistics Review, 1975-2016, National Cancer Institute. Bethesda; 2019. https://seer.cancer. 
gov/csr/1975_2016/ based on November 2018 SEER data submission, posted to the SEER web site, April 2019

3. Lim DH, Maher E. DNA methylation: a form of epigenetic control of gene expression. Obstet Gynaecol. 2010;12:6.

4. Sharif J, Koseki H. Hemimethylation: DNA's lasting odd couple. Science. 2018;359(6380):1102-3.

5. Ehrlich M, Lacey M. DNA hypomethylation and hemimethylation in cancer. Adv Exp Med Biol. 2013;754:31-56.

6. Li E, Zhang Y. DNA methylation in mammals. Cold Spring Harb Perspect Biol. 2014;6(5):a019133.

7. Shao C, Lacey M, Dubeau L, Ehrlich M. Hemimethylation footprints of DNA demethylation in cancer. Epigenetics. 2009;4(3):165-75.

8. Xu C, Corces VG. Nascent DNA methylome mapping reveals inheritance of hemimethylation at CTCF/cohesin sites. Science. 2018;359(6380):1166-70

9. Sun S, Lee YR, Enfield B. Hemimethylation patterns in breast Cancer cell lines. Cancer Informat. 2019;18:1176935119872959.

10. Sun X, Han Y, Zhou L, Chen E, Lu B, Liu Y, Pan X, Cowley AW Jr, Liang $\mathrm{M}, \mathrm{Wu} \mathrm{Q}$, et al. A comprehensive evaluation of alignment software for reduced representation bisulfite sequencing data. Bioinformatics. 2018; 34(16):2715-23.

11. Harris EY, Ponts N, Le Roch $K G$, Lonardi S. BRAT-BW: efficient and accurate mapping of bisulfite-treated reads. Bioinformatics. 2012;28(13):1795-6.

12. Sun S, Li P. HMPL: a pipeline for identifying Hemimethylation patterns by comparing two samples. Cancer Informat. 2015;14(Suppl 2):235-45.

13. Wilcoxon F. Individual comparisons by ranking methods. Biom Bull. 1945; 1(6):80-3.

14. Subramanian A, Tamayo P, Mootha VK, Mukherjee S, Ebert BL, Gillette MA, Paulovich A, Pomeroy SL, Golub TR, Lander ES, et al. Gene set enrichment analysis: a knowledge-based approach for interpreting genome-wide expression profiles. Proc Natl Acad Sci U S A. 2005;102(43):15545-50.

15. GeneCards - Gene Database (www.genecards.org). Accessed 21 Feb 2021.

16. Doherty R, Couldrey C. Exploring genome wide bisulfite sequencing for DNA methylation analysis in livestock: a technical assessment. Front Genet. 2014;5:126.

17. Rodriguez N, Pelaez A, Barderas R, Dominguez G. Clinical implications of the deregulated TP73 isoforms expression in cancer. Clin Transl Oncol. 2018; 20(7):827-36.

18. Yao Z, Di Poto C, Mavodza G, Oliver E, Ressom HW, Sherif ZA. DNA methylation activates TP73 expression in hepatocellular carcinoma and gastrointestinal Cancer. Sci Rep. 2019;9(1):19367.

19. Ye H, Guo X. TP73 is a credible biomarker for predicting clinical progression and prognosis in cervical cancer patients. Biosci Rep. 2019;39(8):1-8.

20. Hollstein PE, Shaw RJ. GNAS shifts metabolism in pancreatic cancer. Nat Cell Biol. 2018;20(7):740-1.

21. Idziaszczyk S, Wilson CH, Smith CG, Adams DJ, Cheadle JP. Analysis of the frequency of GNAS codon 201 mutations in advanced colorectal cancer. Cancer Genet Cytogenet. 2010;202(1):67-9.

22. Ikuta K, Seno H, Chiba T. Molecular changes leading to gastric cancer: a suggestion from rare-type gastric tumors with GNAS mutations. Gastroenterology. 2014;146(5):1417-8.

23. Jin X, Zhu L, Cui Z, Tang J, Xie M, Ren G. Elevated expression of GNAS promotes breast cancer cell proliferation and migration via the PI3K/AKT/ Snail1/E-cadherin axis. Clin Transl Oncol. 2019;21(9):1207-19.

24. Tominaga E, Tsuda H, Arao T, Nishimura S, Takano M, Kataoka F, Nomura H, Hirasawa A, Aoki D, Nishio K. Amplification of GNAS may be an independent, qualitative, and reproducible biomarker to predict progression-free survival in epithelial ovarian cancer. Gynecol Oncol. 2010; 118(2):160-6.

25. Gan RH, Wei H, Xie J, Zheng DP, Luo EL, Huang XY, Xie J, Zhao Y, Ding LC, $\mathrm{Su} \mathrm{BH}$, et al. Notch1 regulates tongue cancer cells proliferation, apoptosis and invasion. Cell Cycle. 2018;17(2):216-24.

26. Zeng JS, Zhang ZD, Pei L, Bai ZZ, Yang Y, Yang H, Tian QH. CBX4 exhibits oncogenic activities in breast cancer via Notch1 signaling. Int J Biochem Cell Biol. 2018;95:1-8.

27. Kamburov A, Pentchev K, Galicka H, Wierling C, Lehrach H, Herwig R. ConsensusPathDB: toward a more complete picture of cell biology. Nucleic Acids Res. 2011;39(Database issue):D712-7.

28. Kamburov A, Stelzl U, Lehrach H, Herwig R. The ConsensusPathDB interaction database: 2013 update. Nucleic Acids Res. 2013;41(Database issue):D793-800
29. Kamburov A, Wierling C, Lehrach H, Herwig R. ConsensusPathDB--a database for integrating human functional interaction networks. Nucleic Acids Res. 2009;37(Database issue):D623-8.

30. Redmond KL, Crawford NT, Farmer H, D'Costa ZC, O'Brien GJ, Buckley NE, Kennedy RD, Johnston PG, Harkin DP, Mullan PB. T-box 2 represses NDRG1 through an EGR1-dependent mechanism to drive the proliferation of breast cancer cells. Oncogene. 2010;29(22):3252-62.

31. Shajahan-Haq AN, Boca SM, Jin L, Bhuvaneshwar K, Gusev Y, Cheema AK, Demas DD, Raghavan KS, Michalek R, Madhavan S, et al. EGR1 regulates cellular metabolism and survival in endocrine resistant breast cancer. Oncotarget. 2017:8(57):96865-84.

32. Wenzel K, Daskalow K, Herse F, Seitz S, Zacharias U, Schenk JA, Schulz H, Hubner N, Micheel B, Schlag PM, et al. Expression of the protein phosphatase 1 inhibitor KEPI is downregulated in breast cancer cell lines and tissues and involved in the regulation of the tumor suppressor EGR1 via the MEK-ERK pathway. Biol Chem. 2007;388(5):489-95.

33. Yang $M$, Teng W, Qu Y, Wang H, Yuan Q. Sulforaphene inhibits triple negative breast cancer through activating tumor suppressor Egr1. Breast Cancer Res Treat. 2016;158(2):277-86.

34. Kong C, Zhan B, Piao C, Zhang Z, Zhu Y, Li Q. Overexpression of UNC5B in bladder cancer cells inhibits proliferation and reduces the volume of transplantation tumors in nude mice. BMC Cancer. 2016; 16(1):892.

35. Liu J, Kong CZ. Expressions of netrin-1 and UNC5B in prostate cancer and their clinical significance. Zhonghua Nan Ke Xue. 2013;19(12):1072-6.

36. Liu J, Zhang Z, Li ZH, Kong CZ. Clinical significance of UNC5B expression in bladder cancer. Tumour Biol. 2013:34(4):2099-108.

37. Okazaki S, Ishikawa T, lida S, Ishiguro M, Kobayashi H, Higuchi T, Enomoto M, Mogushi K, Mizushima H, Tanaka H, et al. Clinical significance of UNC5B expression in colorectal cancer. Int J Oncol. 2012;40(1):209-16.

38. Feng $Y H$, Su YC, Lin $S F$, Lin PR, Wu CL, Tung $C L$, Li CF, Shieh GS, Shiau AL. Oct4 upregulates osteopontin via Egr1 and is associated with poor outcome in human lung cancer. BMC Cancer. 2019;19(1):791.

39. Sun M, Nie FQ, Zang C, Wang Y, Hou J, Wei C, Li W, He X, Lu KH. The Pseudogene DUXAP8 promotes non-small-cell lung Cancer cell proliferation and invasion by epigenetically silencing EGR1 and RHOB. Mol Ther. 2017; 25(3):739-51.

40. Thiebault K, Mazelin L, Pays L, Llambi F, Joly MO, Scoazec JY, Saurin JC, Romeo $G$, Mehlen P. The netrin-1 receptors UNC5H are putative tumor suppressors controlling cell death commitment. Proc Natl Acad Sci U S A. 2003;100(7):4173-8.

41. Xu H, Han Y, Liu B, Li R. UNC-5 homolog B (UNC5B) is one of the key downstream targets of N-a-Acetyltransferase 10 (Naa10). Sci Rep. 2016;6:1-7.

42. Rao RC, Dou Y. Hijacked in cancer: the KMT2 (MLL) family of methyltransferases. Nat Rev Cancer. 2015;15(6):334-46.

43. Bagegni N, Thomas S, Liu N, Luo J, Hoog J, Northfelt DW, Goetz MP, Forero A, Bergqvist M, Karen J, et al. Serum thymidine kinase 1 activity as a pharmacodynamic marker of cyclin-dependent kinase $4 / 6$ inhibition in patients with early-stage breast cancer receiving neoadjuvant palbociclib. Breast Cancer Res. 2017;19(1):123.

44. Zhang C, Song C, Liu T, Tang R, Chen M, Gao F, Xiao B, Qin G, Shi F, Li W, et al. KMT2A promotes melanoma cell growth by targeting hTERT signaling pathway. Cell Death Dis. 2017;8(7):e2940.

45. Huang YC, Lin SJ, Shih HY, Chou CH, Chu HH, Chiu CC, Yuh CH, Yeh TH, Cheng YC. Epigenetic regulation of NOTCH1 and NOTCH3 by KMT2A inhibits glioma proliferation. Oncotarget. 2017;8(38):63110-20.

46. Xiang Y, Zeng H, Liu X, Zhou H, Luo L, Duan C, Luo X, Yan H. Thymidine kinase 1 as a diagnostic tumor marker is of moderate value in cancer patients: a meta-analysis. Biomed Rep. 2013;1(4):629-37.

47. Weagel EG, Burrup W, Kovtun R, Velazquez EJ, Felsted AM, Townsend MH, Ence ZE, Suh E, Piccolo SR, Weber KS, et al. Membrane expression of thymidine kinase 1 and potential clinical relevance in lung, breast, and colorectal malignancies. Cancer Cell Int. 2018;18:135.

48. Wei YT, Luo YZ, Feng ZQ, Huang QX, Mo AS, Mo SX. TK1 overexpression is associated with the poor outcomes of lung cancer patients: a systematic review and meta-analysis. Biomark Med. 2018;12(4):403-13.

49. Li W, Huang K, Guo H, Cui G. Meis1 regulates proliferation of non-small-cell lung cancer cells. J Thorac Dis. 2014;6(6):850-5.

50. Li Z, Huang H, Chen P, He M, Li Y, Arnovitz S, Jiang X, He C, Hyjek E, Zhang J, et al. Publisher correction: miR-196b directly targets both HOXA9/MEIS1 
oncogenes and FAS tumour suppressor in MLL-rearranged leukaemia. Nat Commun. 2018;9:16192.

51. Li Z, Huang H, Chen P, He M, Li Y, Arnovitz S, Jiang X, He C, Hyjek E, Zhaeng J, et al. miR-196b directly targets both HOXA9/MEIS1 oncogenes and FAS tumour suppressor in MLL-rearranged leukaemia. Nat Commun. 2012;3:688.

52. Imamura T, Morimoto A, Takanashi M, Hibi S, Sugimoto T, Ishii E, Imashuku S. Frequent co-expression of HoxA9 and Meis1 genes in infant acute lymphoblastic leukaemia with MLL rearrangement. Br J Haematol. 2002; 119(1):119-21.

53. Xu C, Corces VG. Resolution of the DNA methylation state of single CpG dyads using in silico strand annealing and WGBS data. Nat Protoc. 2019; 14(1):202-16.

54. Guntrum M, Vlasova E, Davis TL. Asymmetric DNA methylation of $\mathrm{CpG}$ dyads is a feature of secondary DMRs associated with the D|k1/Gtl2 imprinting cluster in mouse. Epigenetics Chromatin. 2017;10:31.

55. Nechin J, Tunstall E, Raymond N, Hamagami N, Pathmanabhan C, Forestier $\mathrm{S}$, Davis TL. Hemimethylation of CpG dyads is characteristic of secondary DMRs associated with imprinted loci and correlates with 5hydroxymethylcytosine at paternally methylated sequences. Epigenetics Chromatin. 2019;12(1):64.

56. Patino-Parrado I, Gomez-Jimenez A, Lopez-Sanchez N, Frade JM. Strandspecific CpG hemimethylation, a novel epigenetic modification functional for genomic imprinting. Nucleic Acids Res. 2017;45(15):8822-34.

57. Cai L, Bai H, Duan J, Wang Z, Gao S, Wang D, Wang S, Jiang J, Han J, Tian Y, et al. Epigenetic alterations are associated with tumor mutation burden in non-small cell lung cancer. J Immunother Cancer. 2019;7(1):198.

58. Bjaanaes MM, Fleischer T, Halvorsen AR, Daunay A, Busato F, Solberg S, Jorgensen L, Kure E, Edvardsen H, Borresen-Dale AL, et al. Genome-wide DNA methylation analyses in lung adenocarcinomas: association with EGFR, KRAS and TP53 mutation status, gene expression and prognosis. Mol Oncol. 2016;10(2):330-43.

59. Integrative Onco Genomics (www.intogen.org). Accessed 21 Feb 2021.

60. Liu SH, Shen PC, Chen CY, Hsu AN, Cho YC, Lai YL, Chen FH, Li CY, Wang SC, Chen M, et al. DriverDBv3: a multi-omics database for cancer driver gene research. Nucleic Acids Res. 2020;48(D1):D863-70.

61. Tian S, Bertelsmann K, Yu L, Sun S. DNA Methylation Heterogeneity Patterns in Breast Cancer Cell Lines. Cancer Informat. 2016;15(Supple 4):1-9.

62. Ehrlich M. DNA hypomethylation in cancer cells. Epigenomics. 2009; (2): 239-59.

63. Pedersen BS, Schwartz DA, Yang IV, Kechris KJ. Comb-p: software for combining, analyzing, grouping and correcting spatially correlated P-values. Bioinformatics. 2012;28(22):2986-8.

64. Benjamini Y, Hochberg Y. Controlling the false discovery rate: a practical and powerful approach to multiple testing. J R Stat Soc. 1995;57(1):289-300.

65. Kechris KJ, Biehs B, Kornberg TB. Generalizing moving averages for tiling arrays using combined p-value statistics. Stat Appl Genet Mol Biol. 2010;9: Article29.

66. Xu L, Mitra-Behura S, Alston B, Zong Z, Sun S. Identifying DNA methylation variation patterns to obtain potential breast cancer biomarker genes. Int J Biomed Data Mining. 2015;4(1):1-8.

\section{Publisher's Note}

Springer Nature remains neutral with regard to jurisdictional claims in published maps and institutional affiliations.

Ready to submit your research? Choose BMC and benefit from:

- fast, convenient online submission

- thorough peer review by experienced researchers in your field

- rapid publication on acceptance

- support for research data, including large and complex data types

- gold Open Access which fosters wider collaboration and increased citations

- maximum visibility for your research: over $100 \mathrm{M}$ website views per year

At BMC, research is always in progress.

Learn more biomedcentral.com/submissions 